\title{
Spectroscopy of Matter Near Criticality
}

\author{
B. A. Bernevig, D. Giuliano, and R. B. Laughlin \\ Department of Physics, Stanford University, Stanford, California 94305
}

(October 26, 2018)

\begin{abstract}
We propose that the finite-frequency susceptibility of matter near a class of zero-temperature phase transition exhibits distinctive excitonic structure similar to meson resonances.
\end{abstract}

PACS numbers: 05.70.Jk, 68.35.Rh, 71.10.Hf, 11.15.Ha

In this paper we predict a new spectroscopic effect that should occur very generally af quantum phase transitions described by $O(n) \sigma$-modeld

$$
\mathcal{L}=\left|\partial_{\nu} \vec{\sigma}\right|^{2}+\mu|\vec{\sigma}|^{2}-\lambda|\vec{\sigma}|^{4}
$$

These are thought to describe any continuous phase transition from a fully gapped quantum disordered insulator to a state with continuous broken symmetry, such as an antiferromagnet or a superconductor. The effect we predict, shown in Fig. 1, is a sequence of resonances in the susceptibility

$$
\chi_{q}(\omega)=\iint \vec{\sigma}(\vec{r}, t) \cdot \vec{\sigma}(0,0) e^{i(q \cdot r-\omega t)} d r d t
$$

when the system is slightly detuned from criticality. On the insulating side these may be understood physically as bound states of excitons. On the ordered side they may be understood as bound states of Goldstones.

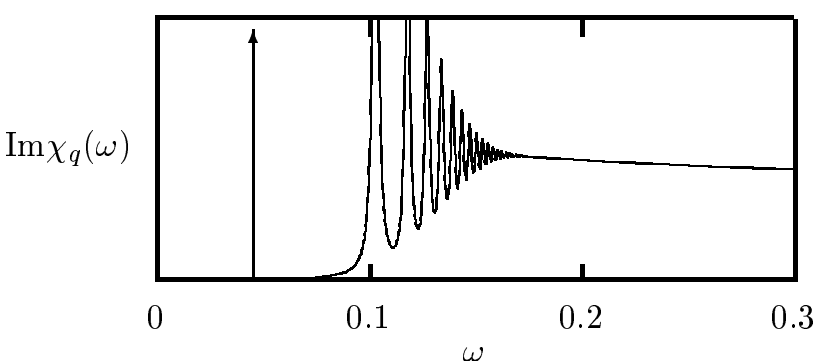

FIG. 1. Proposed spin susceptibility of $\sigma$-model at $q=0$ slightly detuned from its transition on the disordered side.

This effect is important because it is formally similar to a meson spectrum in particle physics, and thus relevant to the larger issue of whether behavior like that of the empty vacuum of space might occur as an emergent phenomenon in ordinary matter. It has been known since the 1970 s that matter undergoing a continuous zerotemperature phase transition is a serious candidate for this, and there is widespread agreement that the lowenergy properties of such matter should be described by simple relativistic field theoriest. However, little is known beyond these basic facts. First-principles theory is impossibly difficult except in a few limiting cases, and all well-characterized quantum phase transitions presently known in the laboratory are either first-order, such as the solidification of ${ }^{4} \mathrm{He}$ under pressure, or disorderdominated. Thus the entire subject of matter undergoing a continuous quantum phase transition in the absence of disorder is largely unexplored, notwithstanding its larger implications 3 .

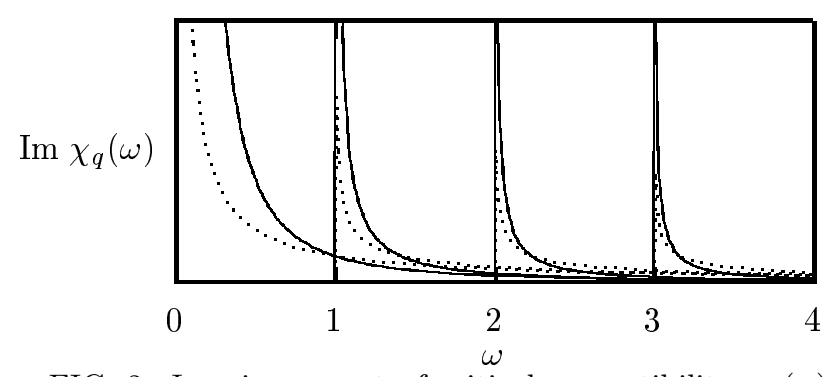

FIG. 2. Imaginary part of critical susceptibility $\chi_{q}(\omega)$ of the $\mathrm{O}(3) \sigma$ model in 2 spatial dimensions as given by Eq. (3) for equally-spaced values of $q$. The dashed curves are the imaginary part of the spin chain susceptibility given by Eq. (4). The limit of small $q$ is assumed in both cases.

Our prediction rests on a physical analogy between critical systems and 1-dimensional spin chains. The standard $\mathrm{O}(3) \sigma$-model has a quantum phase transition as a function of $\mu$. The susceptibility at the phase transitiop has been calculated by $\epsilon$-expansion by various groups 1 and found to have the relativistic form $q^{\eta-2}$, or

$$
\chi_{q}(\omega) \sim \frac{1}{\left[v^{2} q^{2}-\omega^{2}\right]^{1-\eta / 2}},
$$

where $v$ is the speed characterizing the Goldstone mode on the ordered side of the transition and $\eta \simeq 0.031 \pm 0.022$ is the anomalous exponent5. Half-integral spin chains not at a phase transition, on the other hand, have been calculated by exact diagonalization methods to have the strikingly similar susceptibility 6

$$
\chi_{q}(\omega) \sim \frac{1}{\left[v^{2}(q-\pi)^{2}-\omega^{2}\right]^{1 / 2}},
$$

where $v$ is a parameter characteristic of antiferromagnetic exchange. As shown in Fig. 2, in either case one sees a diverging branch cut with a threshold on a relativistic light line. Our idea is that that these spectra are similar because they are the physically the same thing. The spin chain spectra are broad because the lowered dimensionality effectively makes the system critical by preventing 
antiferromagnetism and destabilizing the system toward spin-peirels order. The higher-dimensional critical points have a ground state and excitation spectrum similar to that of the spin chains. However, it has been known since the work of Fadeev and Takhtajand that the breadth of the spin-chain spectra is due ultimately to decay of the injected spin wave into two or more spinons - neutral, spin-1/2 particles not postulated in the underlying equations of motion that may be separated to infinity, do not bind, and do not decay. Thus our idea is that critical points in higher dimension possess new, previously unidentified elementary excitations with integrity analogous to spinons. The pairwise binding of these away from criticality is the basis of the prediction in Fig. 1.

Spinons are particularly easy to write down and explain in the case of the Haldane-Shastry Hamiltonian 8

$$
\mathcal{H}_{H S}=J\left(\frac{2 \pi}{N}\right)^{2} \sum_{\alpha<\beta}^{N} \frac{\vec{S}_{\alpha} \cdot \vec{S}_{\beta}}{\left|z_{\alpha}-z_{\beta}\right|^{2}}
$$

This choice of Hamiltonian is convenient, but not essential, as spinons are believed to be a universal feature of such models, and indeed were originally discovered in the Bethe solution of the Heisenberg chaind 9 . Here $z_{\alpha}=\exp (i 2 \pi \alpha / N)$ is a lattice site on the unit circle expressed as a complex number, and $\vec{S}_{\alpha}$ is a spin- $1 / 2$ spin operator for that site. If the number of sites $N$ is even, then the ground state of this Hamiltonian is the spin singlet 10

$$
\Psi\left(z_{1}, \ldots, z_{N / 2}\right)=\prod_{j<k}^{N / 2}\left(z_{j}-z_{k}\right)^{2} \prod_{j}^{N / 2} z_{j}
$$

where $z_{j}$ denotes the location of the $\mathrm{j}^{\text {th }} \uparrow$ site, all other being down. The energy eigenvalue of this state is $-J \pi^{2}(N+5 / N) / 24$. If $N$ is odd, on the other hand, then the ground state is a doublet, the $\downarrow$ component of which is one of the states

$$
\begin{gathered}
\Psi_{m}\left(z_{1}, \ldots, z_{M}\right)=\sum_{\alpha}\left(z_{\alpha}^{*}\right)^{m} \Phi_{\alpha}\left(z_{1}, \ldots, z_{M}\right) \\
\Phi_{\alpha}\left(z_{1}, \ldots, z_{M}\right)=\prod_{j}^{M}\left(z_{\alpha}-z_{j}\right) \prod_{j<k}^{M}\left(z_{j}-z_{k}\right)^{2} \prod_{j}^{M} z_{j},
\end{gathered}
$$

where $M=(N-1) / 2$. The states $\Psi_{m}$ are propagating spinons of momentum

$$
q_{m}=N \pi / 2-2 \pi(m+1 / 4) / N \quad(\bmod 2 \pi) .
$$

They are eigenstates of the Hamiltonian with eigenvalue

$$
E_{m}=-J\left(\frac{\pi^{2}}{24}\right)\left(N-\frac{1}{N}\right)+\frac{J}{2}\left(\frac{2 \pi}{N}\right)^{2} m(M-m)
$$

Thus the spinon may be thought of as a propagating spin- $1 / 2$ defect caused by the addition of an extra site to an even- $\mathrm{N}$ chain. Combining the momentum and energy expressions, one obtains the dispersion relation

$$
E_{q}=\frac{J}{2}\left[\left(\frac{\pi}{2}\right)^{2}-q^{2}\right] \quad(\bmod \pi)
$$

This tells us that spinons are relativistic particles with Dirac points at $q= \pm \pi / 2$. The states $\Phi_{\alpha}$, which are mathematically equivalent to fractional quantum Hall quasiparticles, describe $\mathrm{a} \downarrow$ spinon at site $\alpha$.

Spinons attract strongly. The spin susceptibility

$$
\operatorname{Im} \chi_{q}(\omega)=\sum_{x}|<x| S_{q-\pi}^{z}|0>|^{2} \delta\left(\omega-E_{x}\right)
$$

of the even-N Haldane-Shastry model, where $\mid 0>$ denotes the ground state, $\mid x>$ and $E_{x}$ denote an excited state and corresponding excitation energy, and

$$
S_{q}^{z}=\sum_{\alpha} z_{\alpha}^{q N / 2 \pi} S_{\alpha}^{z}
$$

is given exactly by 6

$$
\begin{gathered}
\operatorname{Im} \chi_{q}(\omega)=\frac{J}{8 \pi} \int_{-\pi / 2}^{\pi / 2} \int_{-\pi / 2}^{\pi / 2} \frac{\left|k-k^{\prime}\right|}{\sqrt{E_{k} E_{k^{\prime}}}} \\
\times \delta\left(k+k^{\prime}+q\right) \delta\left(E_{k}+E_{k^{\prime}}-\omega\right) d k d k^{\prime}
\end{gathered}
$$

for $-\pi<q<\pi \bmod (2 \pi)$. The physical meaning of this formula is that spinon pairs of total momentum $q$ excited out of the vacuum escape to infinity but also attract each other, in that the wavefunction amplitude for the two particles to coincide is enhanced over the flat 2spinon joint density of states by a matrix element that strongly favors low-energy decays. This effect is crucial for producing the divergence of the branch cut, for the joint density of states is flat.

The strong interaction among spinons cause their field theory to be ambiguous. Writing the Dirac sea of electrons as

$$
\left|\Phi>=\prod_{|k|<\pi / 2, \sigma} c_{k \sigma}^{\dagger}\right| 0>\quad(\bmod 2 \pi),
$$

where $c_{k \sigma}=N^{-1 / 2} \sum_{\alpha} z_{\alpha}^{k N / 2 \pi} c_{\alpha \sigma}$, we find that the even$\mathrm{N}$ ground state of Eq. (6) is exactly $\left|\Psi>=P_{G}\right| \Phi>$, where

$$
P_{G}=\prod_{\alpha}\left(1-c_{\alpha \uparrow}^{\dagger} c_{\alpha \downarrow}^{\dagger} c_{\alpha \downarrow} c_{\alpha \uparrow}\right)
$$

is the Gutzwiller projector, and that the spinon-pair eigenstates are superpositions of the wavefunctions 8 . 10

$$
P_{G} c_{\pi-k \uparrow}^{\dagger} c_{k^{\prime} \downarrow}\left|\Phi>=P_{G} c_{\pi-k^{\prime} \uparrow}^{\dagger} c_{k \downarrow}\right| \Phi>\quad .
$$

Thus spinons have a natural fermi representation in terms of conventional particle and hole excitations of a Dirac sea or metal. However, the representation is degenerate, 
in that an $\uparrow$ spinon has allowed momentum only in the range $-\pi / 2<k<\pi / 2$ may be written either as an $\uparrow$ particle in this range or a $\downarrow$ hole at $\pi-k$. They are thus like Kogut-Susskind fermions, except without the "doubling problem" 11 .

In order to talk concretely about spinon-like excitations potentially present at higher dimensional critical points let us now identify a specific transition that the $\sigma$-model might describe. We propose the Hamiltonian

$$
\begin{gathered}
\mathcal{H}=\sum_{j k \sigma} t_{j k} c_{j \sigma}^{\dagger} c_{k \sigma}+U \sum_{j \sigma \sigma^{\prime}} c_{j \sigma}^{\dagger} c_{j \sigma^{\prime}}^{\dagger} c_{j \sigma^{\prime}} c_{j \sigma} \\
t_{j k}=\left|t_{j k}\right| \exp \left(i \int_{j}^{k} \vec{A} \cdot d \vec{s}\right) \quad \oint_{\text {plaquette }} \vec{A} \cdot d \vec{s}=\pi
\end{gathered}
$$

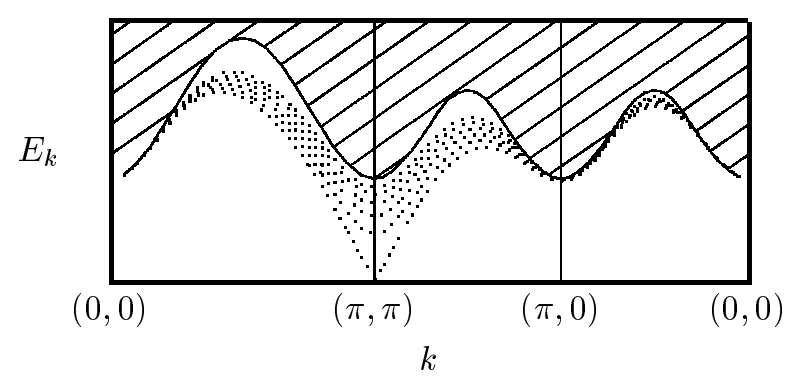

FIG. 3. Spin-1 exciton dispersion defined by Eqs. (4) - (7) for the case of $m_{0}=0.4$ for various values of $U$ approaching the critical value. The solid line is the pair-production threshold.

borrowed from cuprate superconductor theory 12 . This describes electrons moving on a square planar lattice in the presence of a magnetic flux $\pi$ per plaquette, nearneighbor and second-neighbor integrals $t$ and $t^{\prime}$, and an on-site coulomb repulsion $U$. When $U$ is small this system is a conventional insulator with band structure

$$
E_{k}= \pm 2 t \sqrt{\cos ^{2}\left(k_{x}\right)+\cos ^{2}\left(k_{y}\right)+m^{2}}
$$

in Landau gauge $(\vec{A}=\pi y \hat{x})$ with $m=m_{0} \sin \left(k_{x}\right) \sin \left(k_{y}\right)$, $m_{0}=2 t^{\prime} / t$. When $U$ is large it is an ordered antiferromagnet with Heisenberg exchange. The magnetic field in this model is simply a device for constructing a band insulator with one electron per unit cell, and thus enabling the transition to be to a half-integral antiferromagnet.

The parameter $\vec{\sigma}$ in Eq. (1) corresponds to conventional spin, and $\mu$ corresponds roughly to $U$. When $U$ is small, the ladder approximation for the the spin susceptibility is appropriate and gives

$$
\begin{gathered}
\chi_{q}(\omega)=\frac{\chi_{q}^{0}(\omega)}{1+U \chi_{q}^{0}(\omega)} \\
\chi_{q}^{0}(\omega)=\frac{1}{(2 \pi)^{3}} \iint \operatorname{Tr}\left[G_{k}(E) G_{k+q}(E+\omega)\right] d E d k
\end{gathered}
$$

$$
\begin{gathered}
G_{k}^{-1}(E)=E-2 t\left[\cos \left(k_{x}\right) \alpha_{x}+\cos \left(k_{y}\right) \alpha_{y}+m \beta\right] \\
\alpha_{x}=\left[\begin{array}{cc}
1 & 0 \\
0 & -1
\end{array}\right] \quad \alpha_{y}=\left[\begin{array}{ll}
0 & 1 \\
1 & 0
\end{array}\right] \quad \beta=\left[\begin{array}{cc}
0 & -i \\
i & 0
\end{array}\right]
\end{gathered}
$$

The imaginary part of $\chi_{q}(\omega)$ consists of a broad continuum with an edge at the pair-creation threshold and a $\delta$-function representing a spin-1 exciton bound down below this threshold by an amount depending on $U$. This is illustrated in Fig. 3. This exciton at momentum $Q=(\pi, \pi)$ has integrity, i.e. does not decay, even when this calculation is corrected to all orders, because it is the lowest-energy excitation of the system. The energy gap at $(\pi, \pi)$ decreases as $U$ is increased and collapses to zero at the critical value $U_{c}$. As it does so the exciton dispersion becomes relativistic. At the transition the Hartree-Fock equations become unstable to spin density wave formation, and the same ladder sum computed with the broken-symmetry electron propagator then gives three relativistic $\delta$-functions - two light-like spin waves and one massive amplitude mode. The mass of the latter converges to zero at the transition. Both the integrity of the spin waves and their linear dispersion survive correction to all orders by virtue of Goldstone's theorem 13.

Let us now guess, by analogy with Eq. (17) that the spinons that emerge at the transition are Gutzwiller projections of conventional particles and holes of this band structure with $m_{0}$ set to zero. Note that this is not equivalent to assuming that the true electron gap collapses. The system continues to be an insulator across the transition and has no low-energy charged excitations of any kind. This makes the spinons properly relativistic, forces their momenta to add up properly to that of the corresponding spin wave, restricts their momenta to half the Brillouin zone, and causes an $\uparrow$ particle at momentum $\mathrm{k}$ and a $\downarrow$ hole a momentum $(\pi, \pi)-k$ to describe the same spinon.

Let us now simulate the attraction between spinons by re-evaluating Eq. (21) with $m_{0}$ set to zero and $U$ tuned to criticality. This is done in Fig. 4. It may be seen that one properly obtains a continuum with a divergent branch cut on the relativistic light line. As is the case in 1 dimension, the spectrum is a continuum because the spinon pairs always escape to infinity. The threshold is sharp because lower-energy decays are kinematically forbidden. The edge is enhanced because the attractive force between spinons enhances the wavefunction when the particles come together with small relative momentum. But the enhancement is divergent in this case because of criticality. At the phase transition an arbitrarily small perturbation must be able to push the system into the ordered state, i.e. cause the spinons to bind. This forces the susceptibility to be infinite. The exponent of the divergence is 1 in this calculation rather than the correct $2-\eta$, but this simply means that spinons interact by means of a potential with a long-range tail, an effect we have verified numerically. 

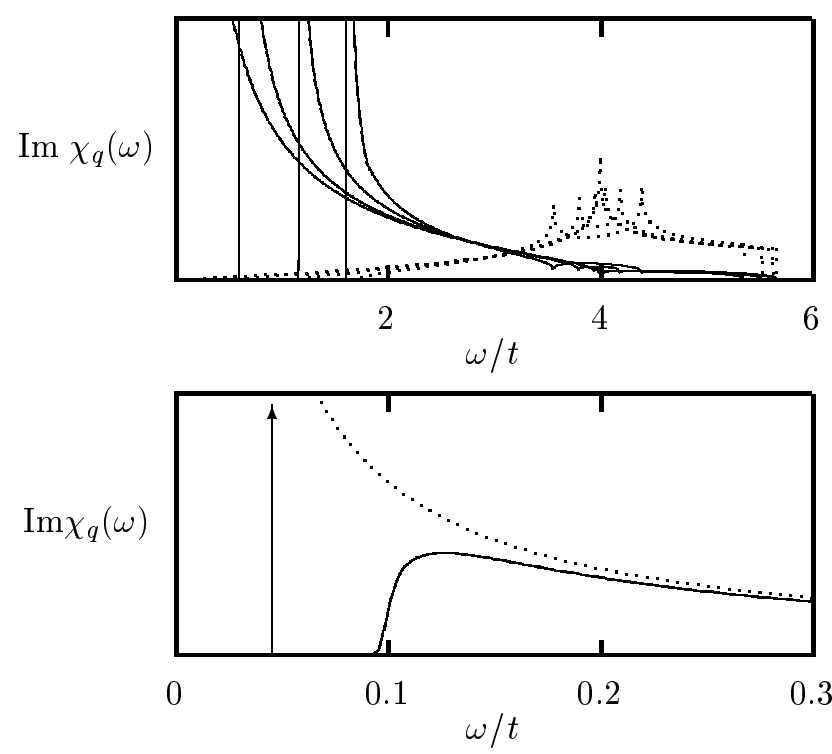

FIG. 4. Top: Imaginary part of the susceptibility $\chi_{q}(\omega)$ defined by Eq. (4) for $m_{0}=0$ and the critical value of $U$ for equally-spaced values of $q$ near $(\pi, \pi)$. The dashed curves are the imaginary part of $\chi_{q}^{0}(\omega)$. Bottom: The same calculation for $q=(\pi, \pi)$ and $m_{0}=0.1$. The dotted curve is the $m_{0} \rightarrow 0$ limit.

Let us now simulate the effect of pushing to the disordered side of the transition by increasing $m_{0}$ slightly keeping $U$ fixed. As shown in Fig. 4, this opens a gap in the continuum and allows for a single bound state, which is the exciton in Fig. 3. The presence of this continuum and its matching the critical susceptibility at high frequencies is required by the principle that a fast measurement should not be able to distinguish a critical system from an almost-critical one. The gap in the continuum at low frequencies is required for the bound state to be sharp. The sharp bound state is required by the integrity of the exciton as the lowest-energy excitation of the disordered phase.

However, a more thoughtful analysis leads to the spectrum of Fig. 1. The continuum threshold in the bottom of Fig. 4 cannot be physically right because spinons are not legitimate elementary excitations of a band insulator. They must cease to exist immediately when the system is pushed away from criticality. The simplest way for this to occur is if the long-range tail of the interaction diverges so as to cause confinement. The result would be a bound-state spectrum more like that of a meson than a traditional exciton. The spectrum in Fig. 1 is calculated assuming that the interaction between the spinons is proportional to $\ln (r)$ rather than a point interaction. We specifically compute

$$
\begin{gathered}
\operatorname{Im} \chi_{q}(\omega) \sim \sum_{n}\left|\psi_{n}(0)\right|^{2} \delta\left(\omega-E_{n}\right) \\
-\frac{1}{2} \nabla^{2} \psi_{n}+\ln (r) \psi_{n}=E_{n} \psi_{n} .
\end{gathered}
$$

The other obvious possibility is a string potential14, expected on the ordered side of the transition, but the result in that case is not much different. In either case one sees a series of bound state resonances that merge into the continuum as the energy scale is raised. Whether these have integrity or decay is determined simply by whether they are above or below the pair-production threshold for the first resonance. These resonances maybe understood physically as bound states of excitons, but a more apt analogy would be with charmonium 15 .

Thus if these resonances are observed experimentally it will suggest strongly that a relativistic gauge theory of some kind, most likely $\mathrm{SU}(2)$ in this case $1 \mathrm{~d}$, can emerge at critical points.

This work was supported primarily by the NSF under grant No. DMR-9813899. Additional support was provided by NASA Collaborative Agreement NCC 2-794 and by NEDO.

${ }^{1}$ M. E. Fisher, Rep. Prog. Phys. 30, 615 (1967).

${ }^{2}$ K. G. Wilson, Rev. Mod. Phys. 55, No.3, 583 (1983).

${ }^{3}$ D. S. Fisher, Physica A 263, 222 (1999).

${ }^{4}$ J. Zinn-Justin, Quantum Field Theory and Critical Phenomena (Oxford, New York, 1993) and references therein.

${ }^{5}$ C. Itzykson and J. M. Drouffe, Statistical Field Theory (Cambridge U. Press, New York, 1989).

${ }^{6}$ F. D. M. Haldane and M. R. Zirnbauer, Phys. Rev. Lett. 71, 4055 (1993); D. A. Tennat et al, Phys. Rev. B 52, 13368 (1995).

${ }^{7}$ L. A. Takhtajan and L. D. Fadeev, Russ. Math. Surveys 34, 11 (1979).

${ }^{8}$ B. S. Shastry, Phys. Rev. Lett. 60, 639 (1988); F. D. M. Haldane, ibid. 60, 635 (1988); ibid. 66, 1529 (1991).

${ }^{9}$ H. A. Bethe, A. Physik 71, 205 (1931).

${ }^{10}$ R. B. Laughlin et al, Field Theory for Low-Dimensional Systems, ed. G. Morandi et al (Springer, Heidelberg, 1999).

${ }^{11}$ J. Kogut and L. Susskind, Phys. Rev. D 11, 395 (1975); K. G. Wilson, ibid., 2445 (1974).

${ }^{12}$ R. B. Laughlin and Z. Zou, Phys. Rev. B 41, 664 (1990); X. G Wen, F. Wilczek and A. Zee, Phys. Rev. B 39, 11413 (1989).

13 J. Goldstone, Nuovo Cimento 19, 154 (1961); P. W. Anderson, Rev. Mod. Phys. 38, 298 (1966).

${ }^{14}$ M. C. Diamantini et al, Phys. Rev. B 5213368 (1995).

${ }^{15}$ T. Applequist et al, Phys. Rev. Lett. 34, 365 (1975).

${ }^{16}$ G. Baskeran, Z. Zou and P. W. Anderson, Solid State Commun. 63, 973 (1987). 